Table 1. Characteristics of patients

\begin{tabular}{lcccc}
\hline & All patients $(\mathrm{n}=26)$ & MTX only $(\mathrm{n}=9)$ & Anti-TNF $\alpha(\mathrm{n}=17)$ & $p$ \\
\hline Age (years) & $51.9 \pm 15.4$ & $60.0 \pm 15.1$ & $47.7 \pm 13.8$ & 0.056 \\
Sex (Female : Male) & $21: 5$ & $6: 3$ & $15: 2$ & 0.331 \\
Disease duration (years) & $6.5 \pm 5.4$ & $7.5 \pm 5.0$ & $5.7 \pm 5.6$ & 0.518 \\
BMI $\left(\mathrm{kg} / \mathrm{m}^{2}\right)$ & $28.3 \pm 7.1$ & $25.2 \pm 5.1$ & $30.1 \pm 7.6$ & 0.109 \\
DAS28 - ESR & $4.27 \pm 2.03$ & $2.17 \pm 0.53$ & $5.79 \pm 1.08$ & $<0.001$ \\
DAS28 -CRP & $3.82 \pm 1.88$ & $1.93 \pm 0.61$ & $5.19 \pm 1.09$ & $<0.001$ \\
\hline
\end{tabular}

increases in erosion volume at $\mathrm{MCH} 2$ and wrist despite low disease activity (Fig. B). In the anti-TNF $\alpha$ group, joint space width and volume of the MCP joints decreased significantly from $\mathrm{BL}$ to $3 \mathrm{M}$ and were positively correlated with erosion volume changes. Although microstructural parameters at the wrist and MCP remained largely unchanged, erosion volume changes were significantly negatively correlated with changes of trabecular BMD in the anti-TNF $\alpha$ group (Fig. C). No significant correlations were observed between HR-pQCT parameters and RAMRIS and DAS scores.

A
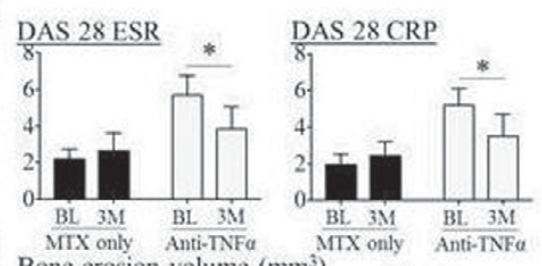

Bone erosion volume (mm
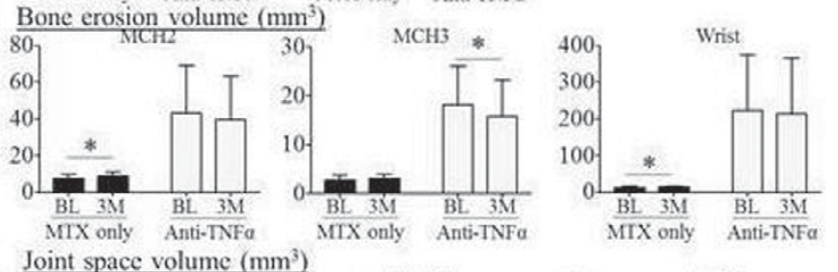
Joint space volume $\left(\mathrm{mm}^{3}\right)$
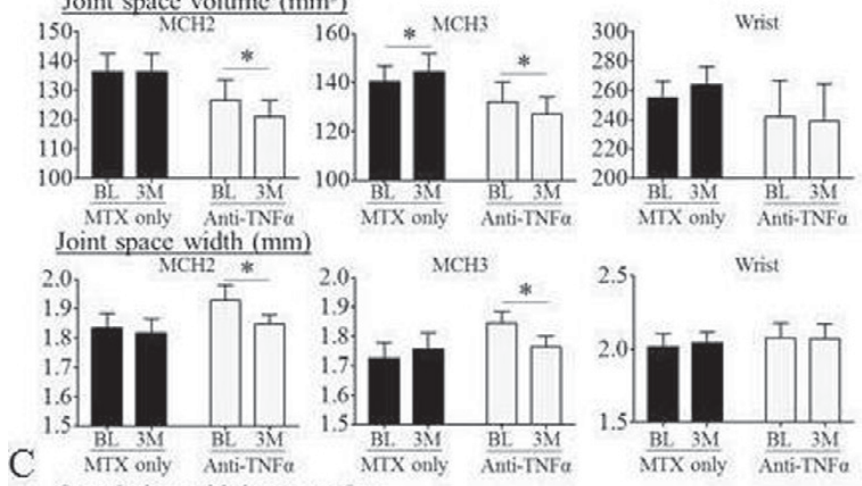

Correlation with bone erosion
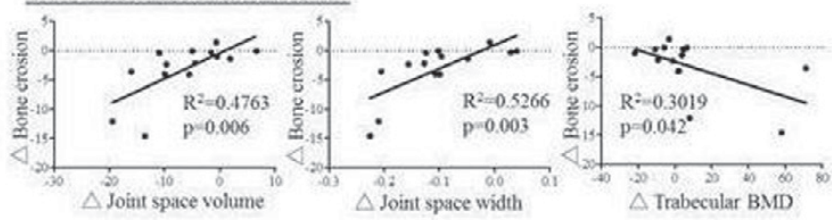

Figure. (A) Comparisons of changes of DAS 28 ESR and CRP from baseline (BL) to 3-months (3M). (B) Comparisons of changes of bone microarchitecture parameters from BL to $3 \mathrm{M}$. * $\mathrm{p}<0.05$; paired $\mathrm{T}$ test.(C) Spearman correlations between changes of erosion volume and each bone parameter in MCP in the anti-TNF $\alpha$ group

Conclusions: We found that anti-TNF $\alpha$ treatment can prevent erosion progression and deterioration of bone microarchitecture within the first 3 months of treatment. HR-pQCT can serve as a sensitive and powerful tool to quantify bone changes and monitor RA treatment even over short time periods.

References:

[1] Barnabe C et al. Nat Rev Rheumatol. 2015.

[2] Tam LS et al. J Rheumatol. 2016.

[3] Moller U et al. Ann Rheum Dis. 2009.

[4] Srikhum W et al. J Rheumatol. 2013.

[5] Yang $\mathrm{H}$ et al. Int J Rheum Dis. 2015.

[6] Burghardt AJ et al. Ann Biomed Eng. 2013.

Acknowledgements: The study was supported by UCB Pharma Inc.

Disclosure of Interest: None declared

DOI: 10.1136/annrheumdis-2017-eular.5321

\section{OP0021 EFFICACY AND SAFETY RESULTS OF A PHASE III STUDY COMPARING FKB327, AN ADALIMUMAB BIOSIMILAR, WITH THE ADALIMUMAB REFERENCE PRODUCT IN PATIENTS WITH ACTIVE RHEUMATOID ARTHRITIS}

R. Alten ${ }^{1}$, J. Glover ${ }^{2}$, N. Matsunaga ${ }^{3}$, D. Chisholm ${ }^{4}$, M. Genovese ${ }^{5} .{ }^{1}$ University Medicine Berlin, Berlin, Germany; ${ }^{2}$ Coephycient Pharmaceutical Consultancy, Guildford, United Kingdom; ${ }^{3}$ Fujifilm Kyowa Kirin Biologics, Chiyoda-ku, Tokyo, Japan; ${ }^{4}$ Fujifilm Kyowa Kirin Biologics, Galashiels, United Kingdom; ${ }^{5}$ Stanford University, Palo Alto, CA, United States

Background: FKB327 is a proposed biosimilar of the adalimumab reference product (ADL). This randomised, double-blind, Phase III study (NCT02260791; FKB327-002) evaluated the therapeutic equivalence of FKB327 and ADL in patients (pts) with active rheumatoid arthritis (RA) inadequately controlled on methotrexate (MTX).

Objectives: To assess the efficacy, safety, pharmacokinetics (PK) and immunogenicity of FKB327 compared with ADL in pts with active RA.

Methods: Pts aged $\geq 18$ years with moderate to severe, active RA for $\geq 3$ months and taking MTX for $\geq 3$ months (10-25 mg/week [wk] stable dose for $\geq 8$ wks) were enrolled. Pts were randomised $1: 1$ to receive FKB327 or ADL (40 mg subcutaneous injection) every 2 wks with continuing MTX, stratified by prior biologic treatment (yes/no) and screening disease activity (Disease Activity Score 28 using C-reactive protein $[\mathrm{DAS} 28-\mathrm{CRP}] \leq 5.1 />5.1)$. The primary efficacy endpoint was American College of Rheumatology (ACR) 20 response rate at Wk 24; equivalence margins of $\pm 13 \%$ were prespecified for a two-sided $95 \%$ confidence interval $(\mathrm{Cl})$ based on $\mathrm{ADL}$ treatment effect in previous studies. Secondary endpoints included ACR20 response rate over time, DAS28-CRP at Wk 24 and over time. Safety was assessed by the incidence/severity of adverse events (AEs) and laboratory abnormalities.

Results: 730 pts from 105 sites in 12 countries were randomised; 728 pts were treated with either FKB327 $(n=366)$ or ADL $(n=362)$. Demographics and baseline RA characteristics were comparable between the groups, with overall mean age of 55.3 years, mean DAS28-CRP score of 6.1 and mean MTX dose of $15.7 \mathrm{mg} / \mathrm{wk}$. ACR20 response rates at Wk 24 (full analysis set) were comparable between the FKB327 (270/363; 74.4\%) and ADL groups (271/358; 75.7\%). 95\% Cls for treatment difference (FKB327 - ADL: -7.6 to 5.0 ) fell within the $\pm 13 \%$ equivalence margins, meeting the primary study objective. ACR20 response rates over time overlapped during Wks 4-24 (Figure 1). Least squares mean DAS28-CRP at Wk 24 were 3.43 on FKB327 and 3.42 on ADL and were equivalent using prespecified margins; changes in scores over time were also comparable. The proportions of pts reporting treatment-emergent AEs (TEAEs) on FKB327 or ADL were similar (FKB327 55.5\% vs ADL 61.6\%); AEs were mainly mild or moderate. The most common TEAEs $(\geq 5 \%)$ were nasopharyngitis $(7.1 \%$ vs $8.0 \%)$ and upper respiratory tract infection (3.6\% vs $5.0 \%$ ). Incidences of serious AEs were similar $(4.1 \%$ vs $5.2 \%)$. One death due to treatment-related disseminated tuberculosis (TB) was reported in the FKB327 group; reports of active TB were slightly higher with ADL (1 vs 3 pts). Mean serum trough concentration-time profiles of the two groups were comparable. The prevalence and titres of antidrug antibodies (ADA) were similar, including at final sampling ( $57.9 \%$ vs $55.8 \%)$.

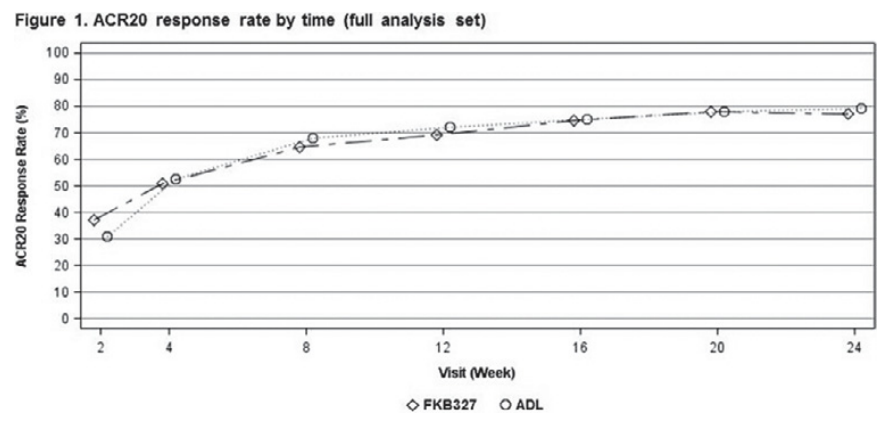

Figure 1

Conclusions: The study met its prespecified equivalency endpoint for ACR20 response rate at Wk 24, with comparable rates over time. Safety profiles, ADA formation and PK were also similar, supporting the comparability of FKB327 and $A D L$ in pts with active RA.

Disclosure of Interest: R. Alten Consultant for: Fujifilm Kyowa Kirin Biologics Co., Ltd, J. Glover Consultant for: Fujifilm Kyowa Kirin Biologics Co., Ltd, N. Matsunaga Employee of: Fujifilm Kyowa Kirin Biologics Co., Ltd, D. Chisholm Employee of: Fujifilm Kyowa Kirin Biologics Co., Ltd, M. Genovese Consultant for: Fujifilm Kyowa Kirin Biologics Co., Ltd, Abbvie, Amgen, Boehringer Ingelheim, Pfizer, Samsung Bioepis

DOI: 10.1136/annrheumdis-2017-eular.2220 